\title{
Optimal Method for Exoplanet Detection by Spectral and Angular Differential Imaging
}

\author{
A. Cornia ${ }^{1,2, a}$, L. M. Mugnier ${ }^{1}$, M. Carbillet ${ }^{3}$, J.-F. Sauvage ${ }^{1}$, A. Boccaletti $^{2}$, N. Védrenne ${ }^{1}$, D. \\ Mouillet $^{4}$, G. Rousset ${ }^{2}$, and T. Fusco ${ }^{1}$ \\ 1 ONERA de Châtillon - DOTA, Unité Haute Résolution Angulaire \\ 2 Observatoire de Meudon - LESIA \\ 3 Université de Nice - Laboratoire Hippolyte Fizeau \\ 4 Laboratoire d'Astrophysique de l'Observatoire de Grenoble
}

\begin{abstract}
In the context of the SPHERE planet finder project, we further develop a recently proposed method, based on detection theory, for the efficient detection of planets using spectral and angular differential imaging. The proposed method uses the fact that with the SPHERE instrument the eld rotates during the night, and that at each acquisition time, two images are recorded by the IRDIS instrument in two different spectral channels. The method starts with the appropriate combination of images recorded at different times, and potentially in different spectral channels, into so-called pseudo-data. It then uses jointly all these pseudo-data in a Maximum-Likelihood (ML) framework to detect the position and amplitude of potential companions of the observed star, taking into account the mixture of photon and detector noises. The method is validated on simulated data.
\end{abstract}

\section{Problem statement}

The direct detection of exoplanets from the ground is a very promising field of astronomy today. A goal is the characterization of the physical composition of the exoplanets by a spectral analysis of their emitted and/or reflected light. This observation from the ground is a technological challenge. Indeed, in order to be able to observe a sufficient number of targets, it is required to cope with an intensity ratio (also called contrast) between the star and its planet that may be as high as $10^{6}$ in IR bands at very small angular separations. In particular, our work is focused on the SPHERE project [1]. This instrument, which will be mounted on VLT of ESO in 2011, is the combination between several optical features, all of them optimized toward the final goal, which is exoplanet detection. First of all, the extreme adaptive optics system (XAO) concentrates the light into a coherent Airy pattern, performing a real-time correction of atmospheric turbulence. Then, the coronagraphic stage strongly attenuates the star intensity and therefore significantly reduces the photon noise. In order to reach the detection performance needed to detect a large number of planets, it is mandatory to combine the above-mentioned optical devices to an a posteriori processing of all the data. The main problematic is to disentangle the potential planet signal from the quasi-static speckles, which are due to static aberrations and constitute a major "noise" source. These speckles present the same characteristic angular size as the planet signal, $\lambda / D$. With no more information, it is impossible to discriminate between the speckles and the planet. In order to do so, the SPHERE instrument includes the ability to perform spectral and angular differential imaging. By subtracting the two images taken at different wavelengths, it is possible to eliminate most of residual aberrations [2]. The two wavelengths are chosen in such a way that there is a strong contrast between the planet fluxes at each of them. But yet, this is not sufficient: one needs to develop a sophisticated method of images processing which allows to exploit also the temporal information. On SPHERE, the pupil is stabilized during the night in order to keep the aberrations fixed, while the field rotates, thus making easier to distinguish them from the planet(s). If the static aberrations are not known, but vary slowly during the night, it is possible to eliminate their influence via appropriate subtractions between images taken at different instants (fig. 1). These double (spectral+angular) differences are the data on which our method is to be applied.

\footnotetext{
a e-mail: alberto.cornia@onera.fr
} the original work is properly cited. 

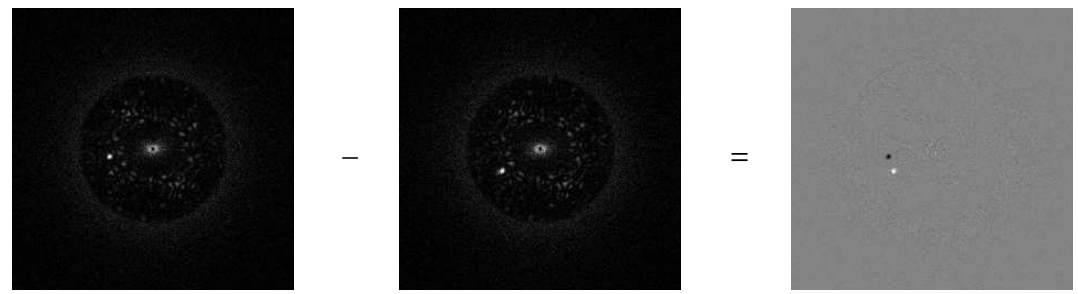

Fig. 1. Left and center: two spectral differences. Right: time difference, in which the star signal has virtually disappeared, leaving only noise and the planet signal (represented by the two peaks, one positive and one negative).

This is the first application of a Maximum-Likelihood method for exoplanet detection via groundbased direct imaging. It represents a refinement of the one proposed in [3]. The improvement introduced are: combination of spectral and angular multi-channel imaging; taking into account the inhomogeneity of noise variance; the positivity constraint of estimated flux; the elaboration of a detection criterion which relies on a statistical basis.

\section{Method}

Each image difference can be written as:

$$
\Delta(\mathbf{r}, k)=\alpha \cdot p\left(\mathbf{r}, k ; \mathbf{r}_{0}\right)+n(\mathbf{r}, k),
$$

where scalar $\alpha$ is the unknown planet intensity, the two-component vector $\mathbf{r}_{0}$ is the unknown planet position at the beginning of the observation (in the first image of the series), $n(\mathbf{r}, k)$ denotes the noise, and $p\left(\mathbf{r}, k ; \mathbf{r}_{0}\right)$ is a synthetic (i.e., noiseless) pattern, which is the precomputed theoretical planet signature (PS) for a planet at an assumed $\mathbf{r}_{0}$ initial position. The PS of index $k$ is simply the difference of two theoretical noiseless planet images (i.e., PSF's) suitably positioned in the field and corresponding to the field rotations at the two instants linked to the index $k$. It can be seen as the space and time-varying PSF of our new data $\Delta(\mathbf{r}, k)$. The maximum likelihood approach consists in searching for $\left(\hat{\mathbf{r}}_{0}, \hat{\alpha}\right)$ that maximize the likelihood $L\left(\mathbf{r}_{0}, \alpha\right)$. In the following we assume that the noise is non-homogeneous (i.e., nonstationary), Gaussian, white both in time $k$ and in space $\mathbf{r}$, and with variance $\sigma^{2}(\mathbf{r}, k)$. This assumption is reasonable and allows us to take into account both the photon and the detector noises. In a Maximum Likelihood approach, the anti-logarithm of the likelihood can be written as follows:

$$
J\left(\mathbf{r}_{0}, \alpha\right)=-\ln L\left(\mathbf{r}_{0}, \alpha\right)=\sum_{k} \sum_{\mathbf{r}} \frac{\left|\Delta(\mathbf{r}, k)-\alpha p\left(\mathbf{r}, k ; \mathbf{r}_{0}\right)\right|^{2}}{2 \sigma_{b}^{2}(\mathbf{r}, k)} .
$$

It can be shown that the optimal value of $\alpha$ for a given $\mathbf{r}_{0}$, which we will call $\hat{\alpha}\left(\mathbf{r}_{0}\right)$, is analytical, because $J\left(\mathbf{r}_{0}, \alpha\right)$ is quadratic with respect to $\alpha$. We can put a positivity constraint on $\hat{\alpha}$, for physical reasons. If we re-inject this estimator of $\alpha$ in the equation of $J$, we can find an expression of the likelihood which depends on $\mathbf{r}_{0}$ only. The maximum of likelihood on the chosen grid gives the most probable position of the planet.

\section{Detection criterion}

Once the likelihood and flux maps are computed, the main problem is to decide which peaks are true companions and which ones are not. One way to do so is to additionally compute the standard deviation of the estimated flux, $\sigma\left(\hat{\alpha}\left(\mathbf{r}_{0}\right)\right)$, for each possible planet position $\mathbf{r}_{0}$, i.e. to compute how the noise propagates from the images to our flux estimator. A possible detection criterion, which can be 

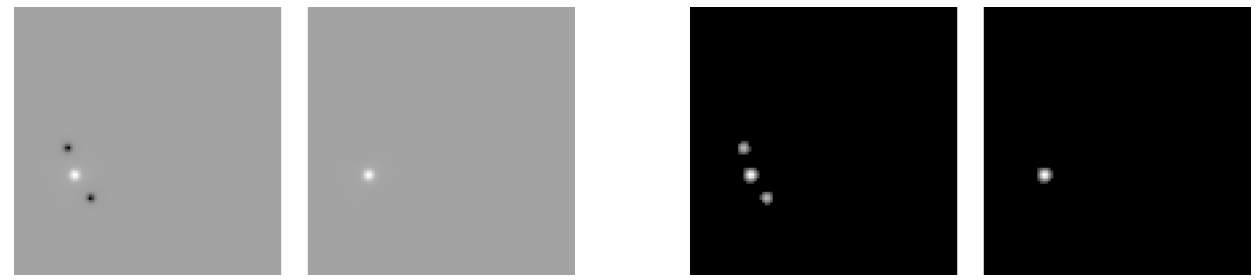

Fig. 2. Estimated flux map, without and with positivity constraint (1st and 2 nd image) and corresponding $J$ maps (3rd and 4th), for a theoretical noiseless case. We can remark the two sidelobes disappearing when the positivity constraint is applied.

linked to the probability of false alarm, is then to decide that all positions where the signal-to-noise ratio (SNR) of the estimated flux, defined as:

$$
\operatorname{SNR}(\hat{\alpha})=\hat{\alpha}\left(\mathbf{r}_{0}\right) / \sigma\left(\hat{\alpha}\left(\mathbf{r}_{0}\right)\right),
$$

is greater than some threshold are true detections.

Interestingly, the SNR of the estimated amplitude is linked very directly to the log-likelihood since it is equal to its square root (except for a constant term). Consequently, maximizing (or thresholding) the likelihood is actually equivalent to maximizing (respectively thresholding) the SNR of the estimated flux.

\section{Pre-treatment}

When performing a difference (spectral or angular) we cannot simply take the value $i_{1}-i_{2}$, because the two images will have different fluxes in the two channels. Thus we have to multiply the second image by a certain factor, which we will call $\gamma$. The optimised spectral and angular difference can be written, respectively, as:

$$
\begin{gathered}
i_{t}^{\prime}=i_{t}^{\lambda_{1}}-\gamma_{t} i_{t}^{\lambda_{2}}, \\
\Delta_{k}=i_{t_{1}(k)}^{\prime}-\gamma_{k}^{\prime} i_{t_{2}(k)}^{\prime},
\end{gathered}
$$

where $i_{t}^{\lambda_{j}}$ is the image taken at the instant $t$ at the wavelength $\lambda_{j}, t_{1}(k)$ and $t_{2}(k)$ are the two instants of observation related to pseudo-image $k$. For the spectral difference, the flux variation is due to the fact that the star spectrum is not perfectly flat around the two considered wavelengths; the time variation is caused by the difference in the quality of AO correction during the night. Furthermore, we can remark that this variation depends greatly on the distance to the centre. So, we optimise the difference only on the annulus we are searching for the planet into.

There are at least two methods to calculate the $\gamma$ coefficient: the most elementary is to equalise the measured fluxes on the considered annulus (so that $\gamma$ is equal to the flux ratio on the annulus), another consists in searching for the value of $\gamma$ which minimises the square of the difference $\left|i_{1}-\gamma i_{2}\right|^{2}$, still on the annulus. Our simulations, as we will see in section 6 , show that the second solution is preferable.

\section{Parametres of simulations}

The method has been tested on realistic data, elaborated simulating SPHERE performances. We put eleven planets in the images, with distances to the star between 4 and $20 \lambda / D$, to test detectivity at different distances. Flux ratio between star and planet is of $10^{6}$. We supposed to have 144 images taken during one night, for a total exposure time of 4 hours (which gives $100 \mathrm{~s}$ per image). We work in $\mathrm{H} 2$ and $\mathrm{H} 3$ bands (1.587 and $1.667 \mu \mathrm{m}$ respectively). Noise is a mixture of photon and detector noise.

The two optimisation techniques described in section 4 have been compared, that is to say the "refined" one (minimisation of the square of the difference on each annulus) and the "rough" one (equalisation of fluxes). 

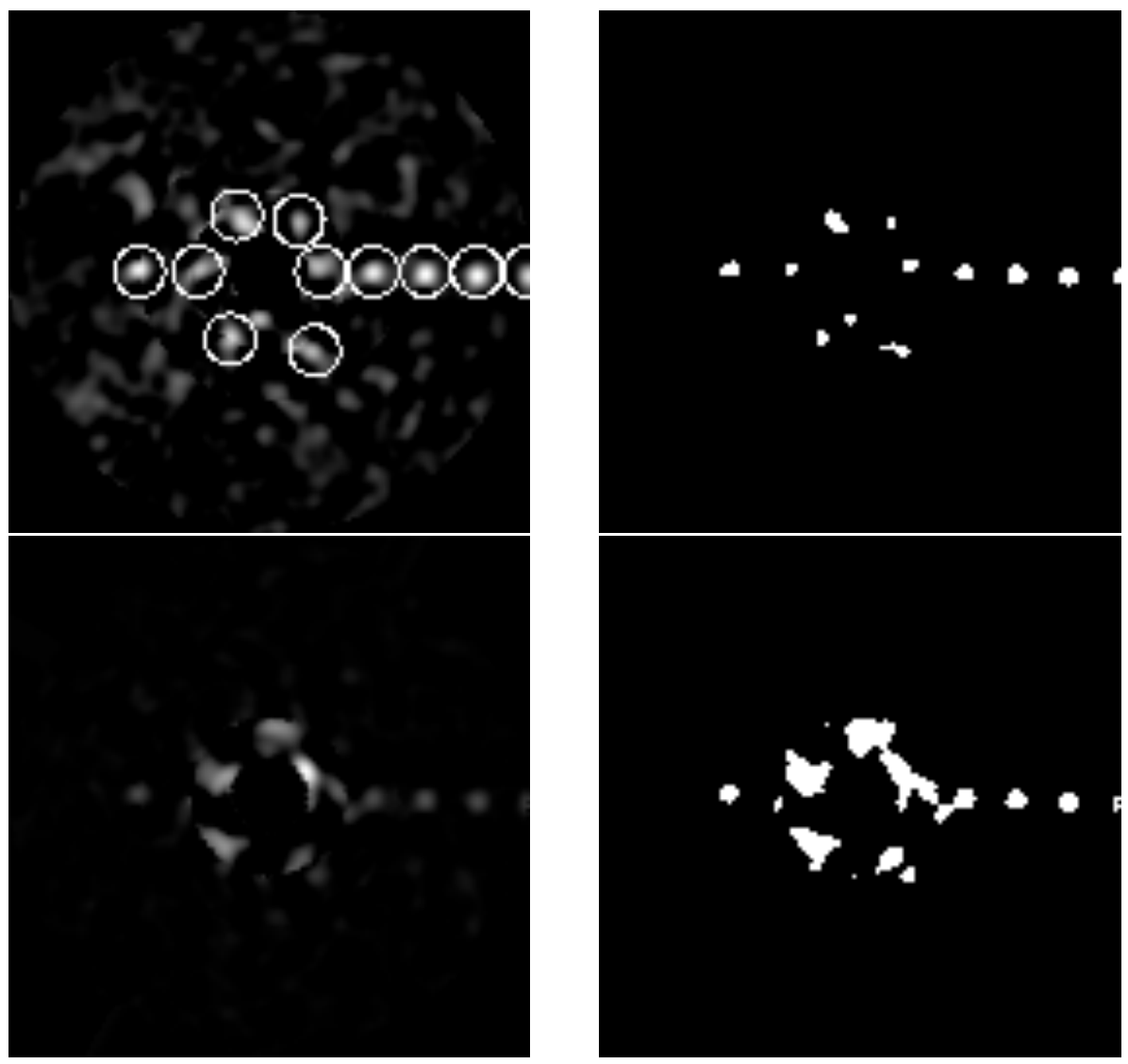

Fig. 3. Top: SNR map (with circles marking the real planet positions) and its thresholded version at $13 \sigma$, obtained with the "refined" optimisation technique. All planets have been detected without false alarms, except a peak very close to the star (at a distance of $3.8 \lambda / D$, that is to say closer than the innermost planet). Bottom: same maps obtained with the "rough" technique. The thresholded map show that in the innermost zone all peaks correspond to false detections.

\section{Results}

Simulations show that joint processing of all images (in both spectral channels) allows detection of planets at a contrast of $10^{6}$ and at a distance of $4 \lambda / D$ to the star. An effective optimisation of the subtractions on different zones is an important factor, as is the knowledge of hypotheses on noise (variance maps). The application of positivity constraint helps to eliminate a part of false alarms.

We also performed tests of robustness of the method in case of mis-centring, using images in which the rotation centre was shifted of a known quantity with respect to the true centre of images. The results of this tests show that detection maps don't change significantly for a centring error of up to 0.5 pixels.

\section{Perspectives}

We intend to refine further on this method by studying in a greater detail the effect of field rotation within each exposure (which causes the signal of planets to spread, especially the outermost) on flux estimation, one of the key parametres to be measured. We also expect to test the algorithm on real images obtained by Subaru and Gemini telescopes. 


\section{References}

1. J.-L. Beuzit, M. Feldt, K. Dohlen, D. Mouillet, P. Puget \& F. Wildi, SPHERE: a Planet Finder instrument for the VLT, in Ground-based and Airborne Instrumentation for Astronomy, 6269 (2008).

2. C. Marois, R. Doyon, D. Nadeau, R. Racine \& G. A. H. Walker, Effects of Quasi-Static Aberrations in Faint Companion Searches, in EAS Publications Series, EAS Publications Series 8 (2003), 233243.

3. L.M. Mugnier, A. Cornia, J.-F. Sauvage, G. Rousset, T. Fusco \& N. Védrenne, Optimal method for exoplanet detection by angular differential imaging, JOSA A 26, Iss. 6(2009), pp. 1326-1334. 